\title{
PTT Advisor: A CDC-supported initiative to develop a mobile clinical laboratory decision support application for the iOS platform
}

Thomas G. Savel, Brian A. Lee, Greg Ledbetter², Sara Brown², Dale LaValley ${ }^{1}$, Julie Taylor ${ }^{3}$, Pam Thompson ${ }^{3}$

1 Informatics R\&D Activity, Public Health Surveillance \& Informatics Program Office, Office of Surveillance, Epidemiology, and Laboratory Services, CDC, Atlanta, GA, 2 CACl International, Inc., Fairfax, VA, 3 Office of Laboratory Science, Policy, and Practice Program Office, Office of Surveillance, Epidemiology, and Laboratory Services, CDC, Atlanta, GA

\section{Abstract}

Objectives: This manuscript describes the development of PTT (Partial Thromboplastin Time) Advisor, one of the first of a handful of iOS-based mobile applications to be released by the US Centers for Disease Control and Prevention (CDC). PTT Advisor has been a collaboration between two groups at CDC (Informatics R\&D and Laboratory Science), and one partner team (Clinical Laboratory Integration into Healthcare Collaborative - CLIHC). The application offers clinicians a resource to quickly select the appropriate follow-up tests to evaluate patients with a prolonged PTT and a normal Prothrombin Time (PT) laboratory result.

Methods: The application was designed leveraging an agile methodology, and best practices in user experience (UX) design and mobile application development.

Results: As it is an open-source project, the code to PTT Advisor was made available to the public under the Apache Software License. On July 6, 2012, the free app was approved by Apple, and was published to their App Store.

Conclusions: Regardless of the complexity of the mobile application, the level of effort required in the development process should not be underestimated. There are several issues that make designing the UI for a mobile phone challenging (not just small screen size): the touchscreen, users' mobile mindset (tasks need to be quick and focused), and the fact that mobile UI conventions/expectations are still being defined and refined (due to the maturity level of the field of mobile application development).

Keywords: Public health, informatics, mobile, clinical decision support, software development

Correspondence: tsavel@cdc.gov

Copyright @2013 the author(s)

This is an Open Access article. Authors own copyright of their articles appearing in the Online Journal of Public Health Informatics. Readers may copy articles without permission of the copyright owner(s), as long as the author and OJPHI are acknowledged in the copy and the copy is used for educational, not-for-profit purposes. 


\section{Introduction}

From 1984 to 2007, The Centers for Disease Control and Prevention (CDC) convened seven institutes on critical issues in clinical laboratory practice.(1) These institutes brought national and international experts together to focus on the role of the clinical laboratory in providing quality testing services for improved patient outcomes. The Clinical Laboratory Integration into Healthcare Collaborative (CLIHC) ${ }^{\mathrm{TM}}$, established by CDC's Division of Laboratory Science and Standards (DLSS), within the Laboratory Science, Policy, and Practice Program Office (LSPPPO), has been addressing some of the recommendations from the past institutes by focusing on important gaps to be filled to optimize the ability of practicing clinicians to effectively utilize laboratory services for better patient care.(2) One of the CLIHC subgroups focused on Diagnostic Algorithms to address clinicians' challenges in laboratory test selection by raising awareness of the complexity of choosing the most appropriate laboratory test for evaluating patients in what appears to be a straightforward clinical setting.

The output of this group has been diagnostic testing algorithms, as well as the recommendation that value could be found in the use of information technology tools to guide clinicians' selection of appropriate laboratory tests. Thus, while the final touches were being placed on the algorithms for patients with abnormal PTT and normal PT values, CDC staff from LSPPPO reached out to CDC's Informatics Research \& Development (R\&D) Laboratory (dry lab) for consultation on the potential implementation of the diagnostic algorithms in a mobile application. $(3,4)$ The R\&D team became active collaborators on the project, and began the development of a prototype decision support app for the iPhone (iOS), PTT Advisor. The prototype goal was to leverage the algorithms as documented in flow charts and turn them into an electronic, interactive, decision support tool for clinical provider use. In other words, the mobile application would be designed to be very intuitive for the users by presenting one question / decision point at a time - and automatically walking them through the algorithm.

\section{Methods}

The creation of a high-quality mobile app is never a trivial task. To facilitate rapid development, an agile development philosophy was adopted. Very early versions of the app were shared in successive iterations with the program staff for feedback, thus allowing for rapid course corrections. In addition, it was crucial that the R\&D team leveraged two very different types of expertise: mobile software development, and user experience design. Both skills were needed to insure the highest-quality mobile app. User requirements specific to clinical providers were prioritized and implemented in quick iterations using feedback from closely involved pathologists (who created the algorithms), and physicians (who used the functionality).

In addition to functional requirements, the design team used industry best practice for core app requirements, such as: intuitive design with easy navigation; rapid, just-in-time error correction; and efficient code design to facilitate multiple changes to the overall algorithm, if needed. Finally, while PTT Advisor is not considered a "Mobile Medical App" from an FDA regulatory authority standpoint, the design team used the emerging FDA draft guidance on mobile medical applications as a source of health and medical-specific requirements for usage, security and privacy.(5) 
The smartphone ecosystem is continuing to mature and evolve at a rapid pace. At the time that this initiative was started, research demonstrated that, within the physician population, use of Apple iPhone/iOS platform was significantly dominant over other platforms, such as Google Android, RIM, Blackberry, Microsoft Windows Phone, or HP WebOS. Survey data collected from June 1, 2010 and February 28, 2011 showed iPhone/iPad with a 90\% share and Android at $6 \%$. (6). Future versions of the app may support additional devices, such as those running the Android operating system, but our initial development efforts were focused on a single mobile device.

Privacy and security are important aspects of health-related apps. To address potential privacy and security concerns, CDC's R\&D team collaborated with security and legal subject matter experts to review the app and examine how it interacted with user data. This application did not need to collect or transmit any information. As a result, the app clearly communicates to users with an initial splash screen that no data entered into the app is stored nor sent outside of the device. All algorithm definitions are stored locally on the device so the app never uses the network to send or receive data.

The application was written in Objective-C using Xcode 4 as the development environment, and Mercurial as the source code management tool. $(7,8)$ PTT Advisor was distributed to beta testers, initially using the 3rd party tool, TestFlight, and then subsequently, using a proprietary mechanism that involved the use of the BetaBuilder open source software package. $(9,10)$ After having the opportunity to experience the application for 2 weeks, the beta testers submitted feedback to CDC staff via email. As it is an open-source project, the code to PTT Advisor was made available to the public under the Apache Software License. $(11,12)$

To support future revisions to the treatment guidance, as well as additional new algorithms, the application was designed with a modular algorithm navigation engine. The engine does not hard code the algorithms in Objective-C, and thus, allows for any type of algorithm to be defined and updated by a non-programmer.

\section{Discussion}

The CDC Informatics R\&D Team gained many insights from this mobile app initiative. Although the clinical decision support algorithms were very well-defined and designed, in the process of implementing them in a structured mobile application, areas of improvement were quickly brought to light, and the algorithms were subsequently modified. Thus, the value of developing a prototype app to obtain valuable insights can not be overstated.

In addition, the user experience design aspect of the mobile app was critical. The app was optimized for rapid decision making at the point of care, as compared to a training or educational tool. Multiple iterations of the user interface were created and tested to find the most intuitive method for "navigating the algorithm." Figure 1 displays the final version of the central component of the mobile application, and emphasizes simplicity (large buttons, clean color schemes, etc.). As is often the case, an application having a "critical feature" can have a significant impact on user adoption. The application displays a simple, tap-oriented screen. A 
question or statement is provided in the top-half of the screen, and large buttons are provided in the bottom-half of the screen so decision options are easy to distinguish and tap. Additional information, provided in the form of a "Footnote" is made available to the user, where applicable, as a button in the upper-right portion of the screen.

Figure 2 displays the "Evaluation Review" feature of PTT Advisor. This feature is accessible at any time and allows the user to review all the selected steps in the algorithm. The feature also allows the user to change any previously completed step in the algorithm, or if no change is necessary, the user can return to the current step. Thus, this innovative feature ensures very efficient use of the clinician's time, obviating the need to start over if a mistake is made. This feature was refined many times until the optimal experience was achieved.

While the app was designed with a goal of a minimal learning curve, a simple Help screen was still provided to answer basic questions. Figure 3 displays the Help screen, which describes how the toolbar (bottom of Figure 1) can be used. The button design, again, focuses on clarity and simplicity.

After multiple iterations with internal stakeholders, the R\&D team ran a beta test with pathologists and practicing physicians to revise the app based on their feedback. The distribution of PTT Advisor to beta testers was not trivial, and did require close communication with the beta testers. As Apple limits the ability to distribute apps outside of the iTunes App Store, our team had to work closely with each beta tester to obtain their device ID and manually install the app for evaluation and testing. On July 6, 2012, the free app was approved by Apple, and was published in their iTunes App Store. (13) 


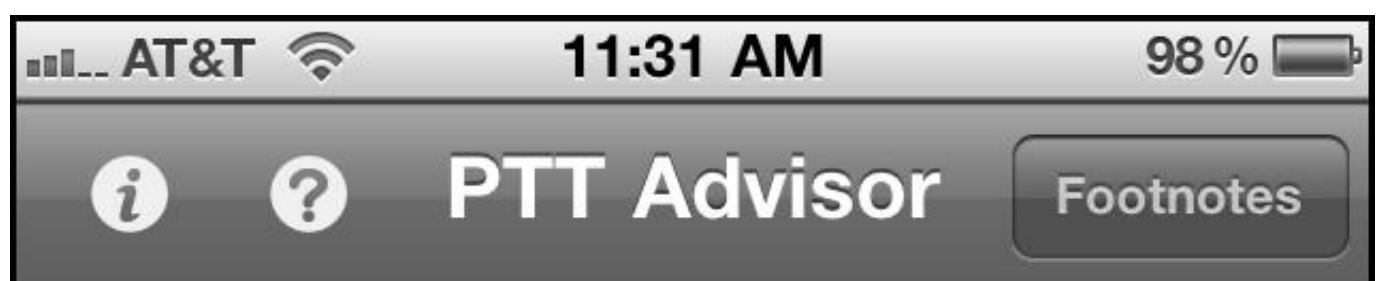

\author{
To Begin, \\ Describe Your Patient
}

\title{
Does the patient have prolonged PTT and normal PT?
}

Yes

\section{No}

Step 1

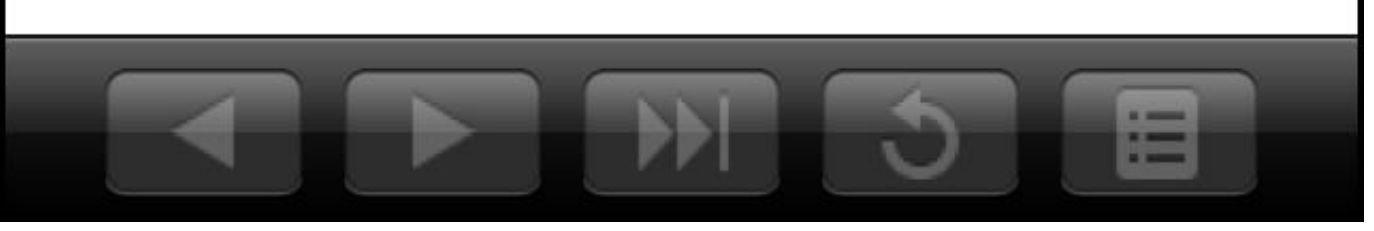

Figure 1. Welcome Screen - PTT Advisor Application 


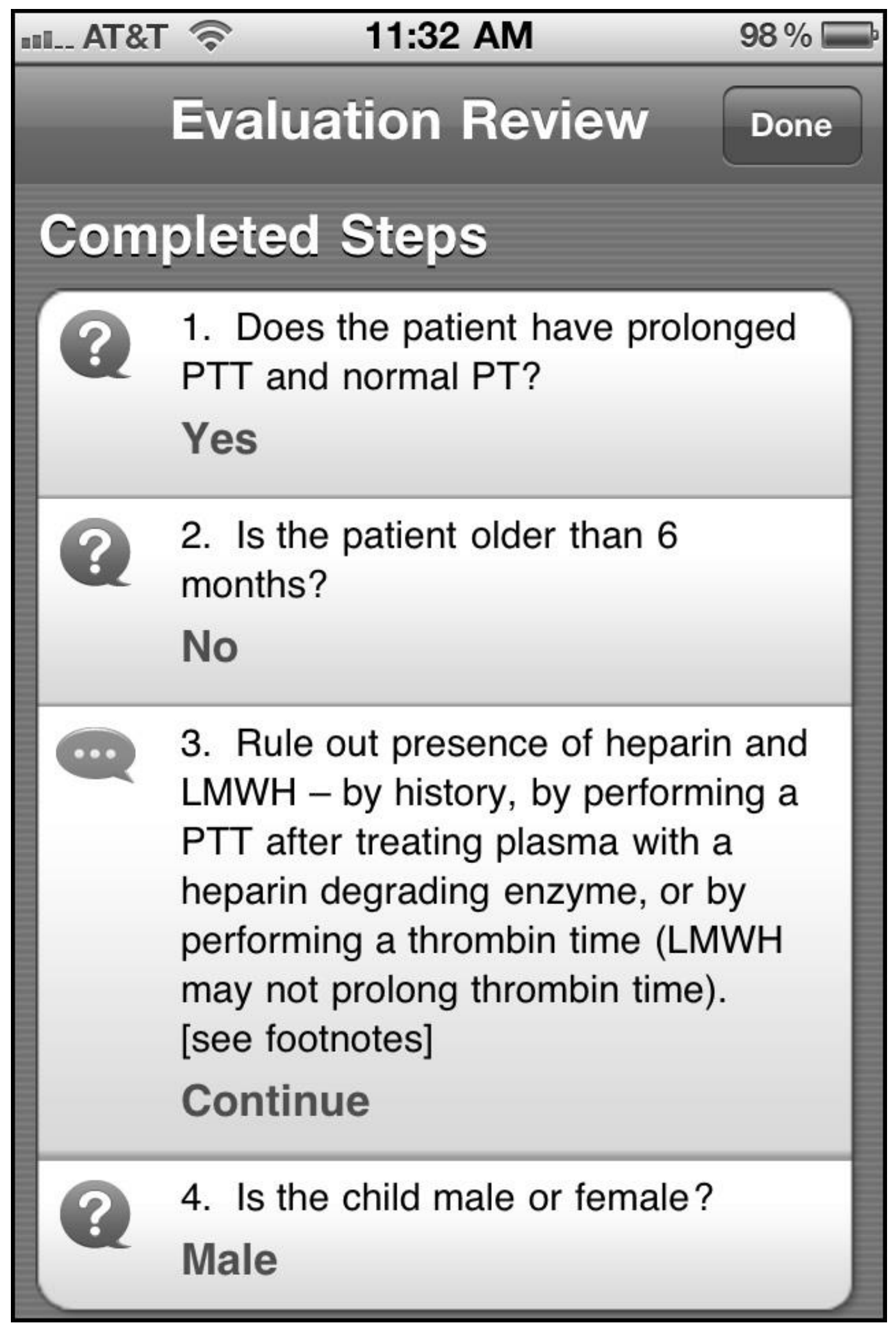

Figure 2. Decision Overview Screen - PTT Advisor Application 


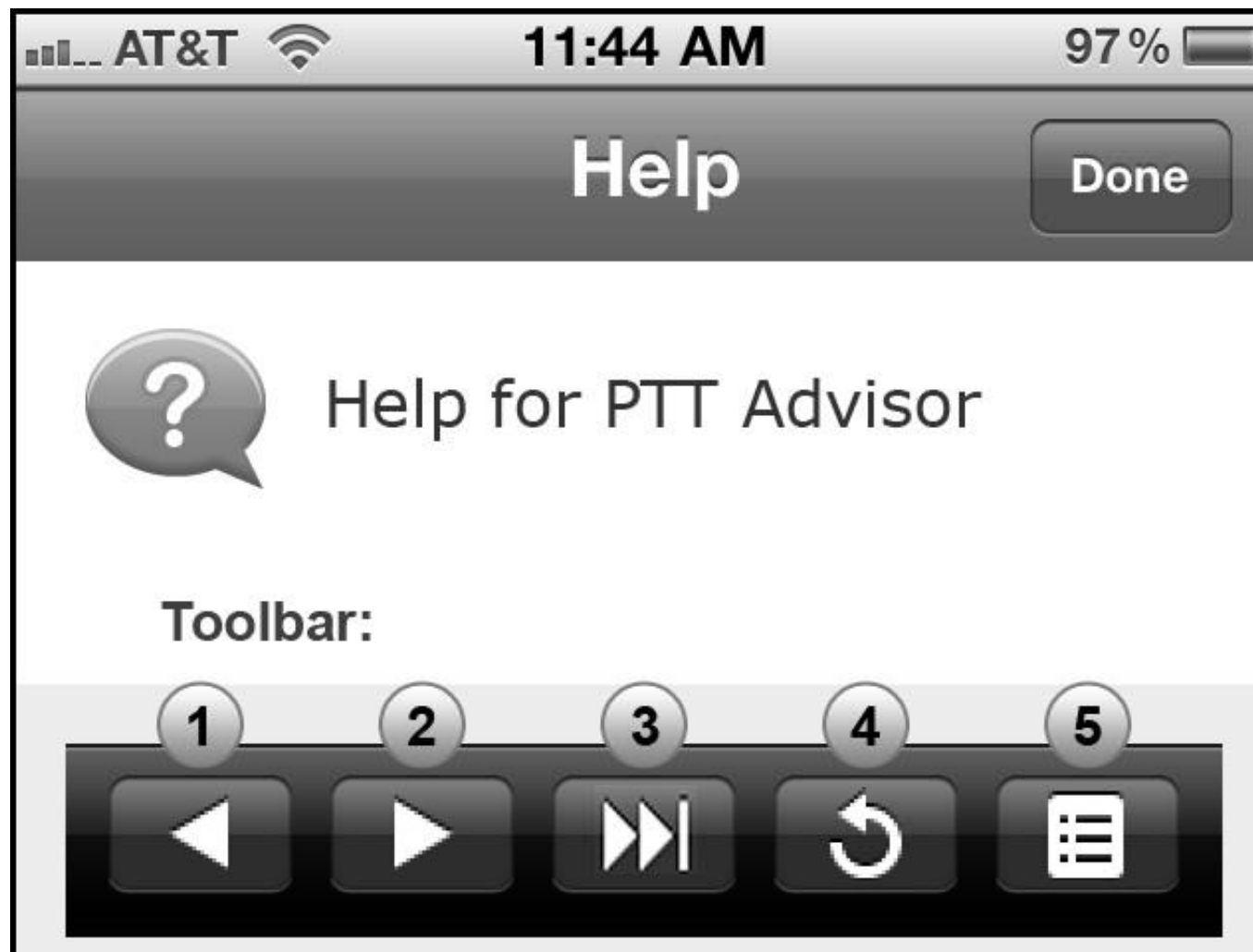

1. Back: Go back one step.

2. Next: Go forward one step.

3. Go to Last: Go to the last step you were presented, but haven't yet responded to.

4. Restart: Restart a patient evaluation.

5. Evaluation Review: Presents a screen that lists the steps and responses so far, including the current step. You may tap a step to edit your response.

Figure 3. Help Screen - PTT Advisor Application 


\section{Conclusion}

Regardless of the complexity of the mobile application, the development process is never easy. There are several issues that make designing the UI for a mobile phone challenging (not just small screen size): the touchscreen, users' mobile mindset (tasks need to be quick and focused), and the fact that mobile UI conventions/expectations are still being defined and refined (due to the maturity level of the field of mobile application development). The extra effort allocated to the UI design will significantly improve user experience.(14)

The goal of User Experience (UX) Design is to provide the user with an optimal experience of the application, including ease-of-use, emotional appeal, features provided, and overall value.(15) It is highly recommended that UX expertise be leveraged in the development of any software (or hardware) solution. The R\&D lab team found that if the software development process uses short iterations with a direct feedback loop to the end users, consistent with the agile development process, it should result in significant time and resource savings. These tight iterations served to not only refine existing developed clinical guidance algorithms, but to more quickly move these changes from design to practicing hands.

With regard to the beta testing and early distribution of a mobile application, is important to note that the complexity of the distribution process will vary, based on the selected distribution tool (e.g., TestFlight), as well as the specific type of license the developers are using (e.g., single developer vs. enterprise). Overall, it is important to factor in adequate time and resources necessary to test the application prior to public launch.

Although PTT Advisor addresses a focused use case, a prolonged PTT and a normal PTT, its design allows for expansion with additional algorithms as they are created, vetted, finalized and released. This is the first in a handful of apps to be released by CDC, in collaboration with its partners, and we look forward to more applications being released in the future.

\section{Acknowledgements}

The authors wish to thank the following individuals for their leadership in the development of the diagnostic algorithm: Michael Laposata, MD, PhD, Marisa B. Marques, MD, and Oxana Tcherniantchouk, MD

\section{Statement on Conflict of Interest}

No conflicts of interest are noted

\section{Corresponding Author}

Thomas G. Savel, MD

Centers for Disease Control and Prevention

1600 Clifton Road, NE, Mail Stop E-55, Atlanta, GA 30329, USA

Email: tsavel@cdc.gov 


\section{References}

[1] CDC's Division of Laboratory Systems - 2007 Institutes http://wwwn.cdc.gov/dls/institutes/ (accessed 16 May 2012).

[2] CDC's Laboratory Science, Policy and Practice Program Office - Clinical Laboratory Integration into Healthcare Collaborative $\left(\right.$ CLIHC) ${ }^{\mathrm{TM}}$ Project Fact Sheets http://www.cdc.gov/osels/lspppo/Laboratory_Medicine_Quality_Improvement/CLIHC_Fact Sheets.html (accessed 16 May 2012).

[3] CDC's Laboratory Science, Policy and Practice Program Office http://www.cdc.gov/osels.lspppo/index.html (accessed 16 May 2012).

[4] CDC's Informatics Research \& Develoment Laboratory http://www.phiresearchlab.org (accessed 10 May 2012).

[5] Draft Guidance for Industry and Food and Drug Administration Staff - Mobile Medical Applications 2011.07.21

http://www.fda.gov/MedicalDevices/DeviceRegulationandGuidance/GuidanceDocuments/uc m263280.htm

[6] Bulletin Healthcare : Physician Mobile Use Grows 45\%; Apple® Dominates Android ${ }^{\mathrm{TM}}$ and Blackberry® http://www.businesswire.com/news/home/20110414005326/en (accessed 5 May 2012).

[7] Apple's Xcode 4 for Developers https://developer.apple.com/xcode/ (accessed 17 May 2012)

[8] Mercurial - source control managmement tool http://mercurial.selenic.com/ (accessed 16 May 2012).

[9] TestFlight - iOS beta testing tool https://testflightapp.com/ (accessed 5 May 2012).

[10] BetaBuilder - a collection of Rake tasks and utilities for managing and publishing Adhoc builds of iOS apps. https://github.com/lukeredpath/betabuilder (accessed 16 May 2012).

[11] Code repository for the open source, mobile application, PTT Advisor https://bitbucket.org/gledbett/anticoagmon/src (accessed 16 May 2012).

[12] Apache License Version 2.0, January 2004. http://www.apache.org/licenses/LICENSE2.0.html (accessed 1 May 2012).

[13] PTT Advisor App in the Apple iTunes App Store. http://itunes.apple.com/us/app/pttadvisor/id537989131?mt=8 Accessed September, 27, 2012.

[14] Clark J. Tapworthy: Designing Great iPhone Apps. Sebastapol, CA: O’Reilly Media Inc. 2010

[15] Garrett J. The Elements of User Experience: User-Centered Design for the Web and Beyond. Berkeley, CA. New Riders Press. Second Edition. 2010. 